\title{
The Complex Quantum and Classical Pseudo-Tachyonic Universe
}

\author{
Dr. Narayan Kumar Bhadra \\ L.S.S.S. High School (H.S.) Gobardanga, North 24 Parganas, West Bengal, India
}

\begin{abstract}
We study a relation between the cosmological singularities in classical and quantum theory, comparing the classical and quantum dynamics in three models possessing the Big-Rip Singularity: the model mainly based on tachyon field and pseudo-tachyon field with respect to the pseudo-space $R_{I}(=i R$, where ' $R$ ' is

the scale factor of the universe and $i=\sqrt{-1}$ ). It was shown that in the pseudo-tachyon model the Hamiltonian is well defined and hence wave function of the universe is not obliged to vanish at the values of the variables, corresponding to the scale factor $R_{I}$ of the appearance of the Big-Rip singularity. There is some kind of a classical-quantum correspondences in the classical cosmological tachyon model exists an infinite oneparameter set of the cosmological evolutions encountering and crossing the Big-Rip singularity. In all other cases-mainly the Big-Brake and Big-Bang singularity in the scalar field model and the Big-Crunch and the Big-Rip singularities in both the tachyon and pseudo tachyon field model we have observed the phenomenon of the quantum avoidance of singularities. It corresponds to the degeneration of the corresponding cosmological trajectories in classical theory. It was shown that a negative pressure may be acquired from Big-Rip Singularity, which is responsible for the Big-Bang singularity and then the expansion of the universe.

Again we study the Hamiltonian formalism for a massive scalar field and particle production with the Gaussian group of energy $S U(6)$, a new type of energy source called latent energy group from the past infinity of the space-like hyper surface and study the de-Sitter Space which assumed with a complex space-time of the scale factor $R+i R_{b}$, where the scale factor $R$ stands for 4-dimensional space time and $\left(i R_{I}\right)$, the internal space of $D$-dimensions, i.e., we consider $d S$-space as $(4+D)$ dimensions with scale factor $R+i R_{I}$. It is compared with Kaluza-Klein Cosmology of $(4+D)$ dimensions.
\end{abstract}

\section{Introduction}

The introduction of the notion of the quantum state of the Gaussian energy group SU(11) [ $\supset \mathrm{SU}(6) \times$ $\mathrm{SU}(5) \times \mathrm{U}(1)]$ of the universe, satisfying the Wheeler-DeWitt equation has stimulated the diffusion of the hypothesis that in the frame-work of quantum cosmology the singularities can disappear in some-sense. Namely, the probability of finding of the universe with the parameters which correspond to a classical cosmological singularity can be equal to zero.

Until the end of nineties almost all the discussions about classical and quantum cosmology of singularities were devoted to the Big-Bang and Big-Crunch singularities, which are characterized by the vanishing value of the cosmological radius. The situation was changed when Edwin Hubble gathered the first evidence of the story in the early 1920's at the Mr. Wilson observatory. By accurately measuring the distances of the nebulae he showed that they were not only separate galaxies, but also that they all had a red-shift, implying that they were all moving away from us. Hubble also observed that the further away the galaxy was, the larger was its red-shift. In fact, the speed of recession of the galaxies, derived from the red-shift, was proportional to their distance. It was soon realized that such an expanding universe was indeed predicted by mathematical models of the universe based on Einstein's general theory of relativity. Such a discovery of the phenomenon of the cosmic acceleration was the starting point for the formulation of cosmological models containing a special type of substance, the so-called dark energy, which, for its specific properties, was considered responsible for the accelerated expansion of the universe, and consequently stimulated the study of the various possible candidates for the role of this substance. The fundamental feature of dark energy which produces an accelerated expansion is that it possesses a negative pressure $p$ such that the strong energy condition $\rho+3 p>0$ is violated. Here, $\rho$ is the energy density. The construction of different cosmological models, has attracted the attention to the fact that other types of cosmological singularities do exist. First of all, one should mention the soft or sudden singularities called Big-Brake cosmological singularity. They occur at a finite value of the scale factor and of its time derivative, and hence of Hubble parameter is equal and exactly to zero and of the energy density, while the second derivative of the scale factor, the first derivative of the Hubble parameter and the pressure are divergent. Another class of singularities should mention the Big-Rip singularity arising in our models where the phantom dark energy also dark matter is present. Under phantom dark-energy one understands the substance whose pressure is negative. Such a singularity is characterized by infinite values of the cosmological radius $R_{I}(=i R)$ and hence of its energy density and pressure of its time derivative, of the 
Hubble parameter derivative. That makes this singularity especially convenient for study. The Big-Rip singularity was first described in this paper, where it has arisen in the context of a particular cosmological model with a pseudo-tachyon field in the space $R_{T}$. Starting from the model of cosmology with Chaplygin and then anti-Chapligin gas filled with universe. According to quantum cosmology we also consider the De-Sitter space having Gaussian energy group SU(11). The $d S$-radiation by Gibbons and Hawking is created due to the presence of the energy group $\mathrm{U}(1)$ by the symmetry breaking of SU(11), there inevitably arise particles that have the characteristics of a magnetic mono-pole and once created they are not destructible as the particles are highly stable and so they would survive as relies to the present epoch.

In the contrast to the early works on the in-out formalism, in this paper we assume the wave-functions for the field created by the energy group $\mathrm{SU}(6)$ and $\mathrm{SU}(5)$ shall play the key role between two space-like hypersurfaces, where $\mathrm{SU}(6)$ in the past-infinity and $\mathrm{SU}(5)$ at an arbitrary time in the future infinity. In cosmological scenarios the Gaussian wave functions on each hyper-surface carry the same information as the Heisenberg field operator. In fact, the in-out formalism and the invariant-operator picture formation are equivalent to each other.

Interestingly, in the planes-co-ordinates of $d S$-space the number of in-vacuum particles carried by the Gaussian wave packet on each hyper-surface exponentially increases. This contrasts with the Gibbons-Hawking radiation which is a thermal and scale factor free distribution in the future infinity. It is shown that the matrix amplitude between the in-vacuum Gaussian wave-packet and the out-vacuum Gaussian wave-packet in the future infinity, however exhibits the Gibbon Hawking radiation. In our model the universe having a complex scale factor, the part of the scale factor of the universe after Big-Crunch to Big-Bang through Big-Rip singularity will have a pseudo-space $R_{I}(=i R)$, where exists a pseudo-energy whose sub-sets are $\mathrm{SU}(11)$ [ $\supset$ $\mathrm{SU}(6) \times \mathrm{SU}(5) \times \mathrm{U}(1)$, where $\mathrm{SU}(5) \supset \mathrm{SU}(3) \times \mathrm{SU}(2) \times \mathrm{U}(1), \mathrm{SU}(3)$, the strong energy group, $\mathrm{SU}(2)$ the weakenergy group and $\mathrm{U}(1)$, the electro-dynamics], which created the negative pressure of the universe from Big-Rip singularities. In the scalar field model we consider the equation of state as $p=\gamma \rho+\frac{A}{\rho^{\alpha}}$, where $\rho$ is the energy density of the universe, and $\gamma, A, \alpha$ all are arbitrary constants. When $\gamma=0$ and $\alpha=1$, i.e., $p=\frac{A}{\rho}(A>0)$ then it was noticed that a very simple cosmological model of the universe, based on the antiChaplygin gas, leads unavoidably to the Big-Brake singularity, which is the Wiggly strings and has got this name in analogy with the Chaplygin gas, which satisfied then an equation of state of the type $p=-\frac{A}{\rho}$ (for $\gamma=$ $0, \alpha=1, A<0$ ) and has acquired a certain importance in cosmology as a candidate for the unification between dark-energy and dark-matter. Starting from Chaplygin gas model with late and early time of the cosmological model and using the technique of reconstruction of the potentials of the pseudo-tachyon field model one can construct the scalar field model reproducing the cosmological evolution. Such a potential was constructed and studied in. In the same the quantum cosmology of the scalar-field model was studied and it was shown that the requirement of the normalizability of the quantum state of the universe, satisfying the Wheeler-DeWitt equation, implies the disappearance of this quantum state at the Big-Brake singularity. Thus, this result looks as confirming the hypothesis that in the frame-work of quantum cosmology the singularities can disappear. Similarly the properties of the solutions of the Wheeler-DeWitt equations for different cosmological models, connected in some way with dark-energy hypothesis as the Big-Rip singularity has given analogous result, for well definiteness of the Hamiltonian by introducing a pseudo-space $R_{I}$. However, the question arises: how general is this phenomenon? To try to answer this question, we shall give a comparative analysis of three cosmological models, encountering the Big-Rip and Big-Brake Singularity in the pseudo-tachyon, tachyon and scalar field model was considered in detail in this paper while the mechanism of the crossing of this singularities was suggested in. Here it was shown that there was a large class of cosmological evolutions crossing the BigBrake singularity as well as Big-Rip singularity. Thus we have some kind of complementarily - the classical dynamics of the tachyon and quantum dynamics of the pseudo-tachyon model with the Big-Rip singularity was studied in detail in and it was shown that this singularity is traversable also the same the classical dynamics for the tachyon model with Big-Brake singularity and on the other hand the quantum dynamics of the model with the scalar field was studied. It is easy to say that there is a correspondence between the classical uniqueness and the quantum elimination of the singularities. Then we study qualitatively the Wheeler-DeWitt equation for the pseudo-tachyon model and we see that the disappearance of the corresponding wave function does not appear. This observation puts the quantum dynamics of the model in correspondence with the classical dynamics where we have a set of trajectories with a non-vanishing measure, which successfully cross the cosmological singularity. 


\section{Graphical Representation of the Space-Time}

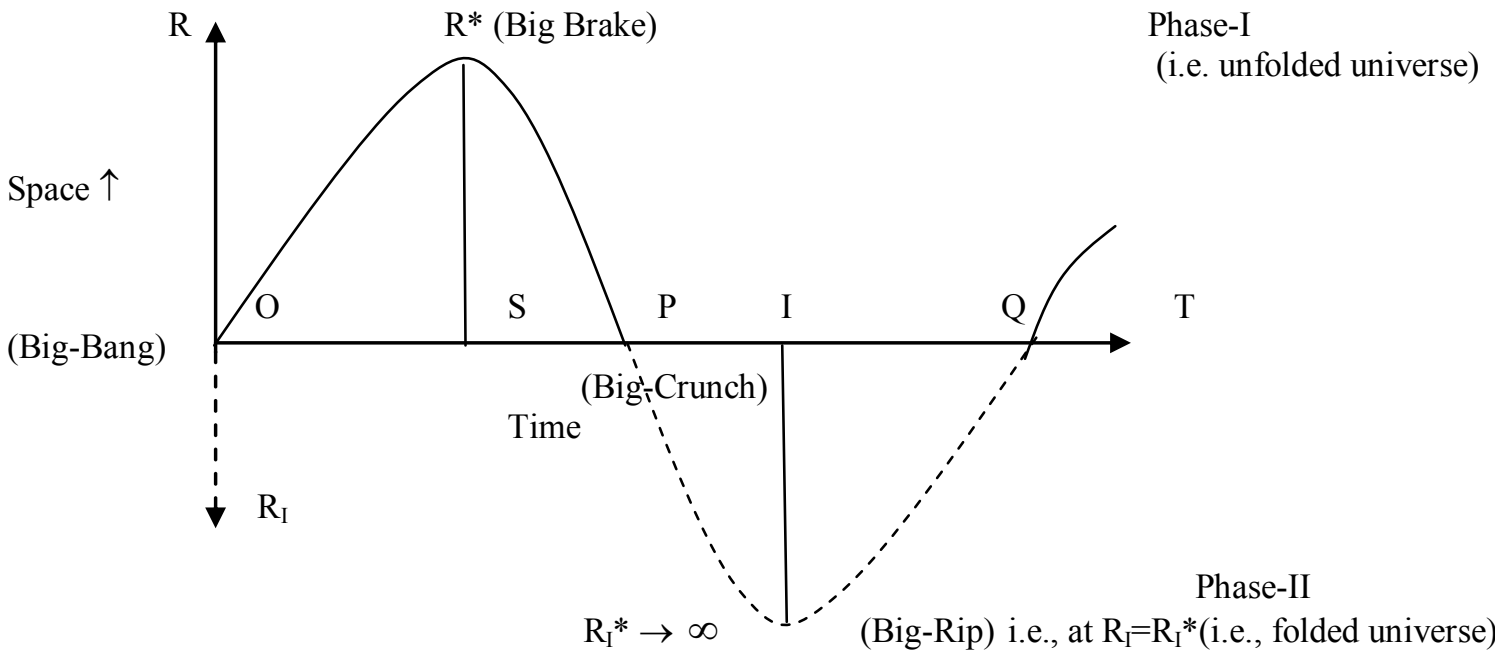

Space-Time Curve

II. The cosmological model with a scalar field potential:

Let us consider a flat Friedmann-Lemaitre-Robertson-Walker universe with metric,

$$
d S^{2}=N^{2}(t) d t^{2}-a^{2}(t) d t^{2}
$$

with the equation of state

$$
p=\gamma \rho+\frac{A}{\rho^{\alpha}} \text { (where } \gamma, A, \alpha \text { are arbitrarily constants). }
$$

The Friedmann equation is

$$
H^{2}=\rho
$$

where the Hubble parameter $H$ is as usual

$$
H \equiv \frac{\dot{R}}{R}
$$

where "dot" means the derivative with respect to the cosmic time $t$. The energy conservation condition is

$$
\dot{\rho}=-3 H(\rho+p)
$$

we have from equation (2) \& (5),

$$
\begin{gathered}
\dot{\rho}=-3 \rho^{1 / 2}\left(\gamma \rho+\frac{A}{\rho^{\alpha}}+\rho\right)=-3 A \rho^{\frac{1}{2}-\alpha}-3(\gamma+1) \rho^{3 / 2} \\
\text { i.e } \frac{H^{2 \alpha+1} d H}{A+(\gamma+1) H^{2 \alpha+2}}=-\frac{3 \dot{R}}{2 R} d t
\end{gathered}
$$

Integrating, we have

$$
\rho(R)=\frac{1}{(\gamma+1)^{1 /(\alpha+1)}}\left[\left(\frac{B}{R}\right)^{3(\gamma+1)(\alpha+1)}-A\right]^{\frac{1}{\alpha+1}}
$$

Again from (3), (4) and (6), we get

$$
\frac{\dot{R}^{2}}{R^{2}}=\frac{1}{(\gamma+1)^{1 /(\alpha+1)}}\left[\left(\frac{B}{R}\right)^{3(\gamma+1)(\alpha+1)}-A\right]^{\frac{1}{\alpha+1}}
$$




$$
\text { i.e., } \quad \dot{R}=\frac{1}{(\gamma+1)^{\frac{1}{2(\alpha+1)}}}\left[\left(\frac{B}{R}\right)^{3(\gamma+1)(\alpha+1)}-A\right]^{\frac{1}{2(\alpha+1)}}
$$

when the cosmological radius tends to the critical value $R^{*}=\frac{B}{1}$, the energy density disappears

$$
A^{\overline{3(\gamma+1)(\alpha+1)}}
$$

and the pressure according to the equation (2) grows indefinitely. Then cosmological time $t=t_{B}$. Again, when the cosmological radius grows indefinitely, i.e., $R \rightarrow \infty$, then the energy density does not disappear and had some parametric value as $\dot{R} \rightarrow \infty$ (i.e., $\dot{R}_{I} \rightarrow \infty$, then $R_{I}=R_{I}^{*}=i R^{*}$ in other phase), then

$$
\rho(R)=\frac{1}{(\gamma+1)^{1 /(\alpha+1)}}[-A]^{\frac{1}{1+\alpha}}
$$

As well as the pressure is then

$$
\begin{aligned}
& p=\frac{\gamma}{(\gamma+1)^{1 /(\alpha+1)}}[-A]^{\frac{1}{1+\alpha}}+A \cdot \frac{1}{(\gamma+1)^{\frac{\alpha}{\alpha+1}}}[-A]^{-\frac{\alpha}{\alpha+1}} \\
& p(R)=\frac{A}{[-A]^{\alpha /(\alpha+1)} \cdot(\gamma+1)^{1 /(\alpha+1)}} \text { is negative for } A<0, \gamma>0, \alpha>0 .
\end{aligned}
$$

Hence, $\quad \rho+3 p=\frac{2 A}{(\gamma+1)^{1 /(\alpha+1)}[-A]^{\alpha /(\alpha+1)}}$

is not real for $A>0, \alpha>0, \gamma>-1$ and $\rho+3 p \ngtr 0$ when $\quad A \ngtr 0$. If $\gamma=0$ and $A<0, \alpha=1$, the universe coincide with the Chaplygin gas model (and pressure is then negative. Also for $\gamma=-1$ and $A<0$, then pressure $p$ will be $-\infty$ and $\rho$ is then $\infty$. Thus the strong energy condition $\rho+3 p>0$ violated due to the different value of the constant parameters, we have from the equation (7),

$$
\ddot{R}=-\frac{1}{2(\gamma+1)^{\frac{1}{(\alpha+1)}} R^{3 \gamma+2}} \cdot \frac{2 A R^{3(\gamma+1)(\alpha+1)}+(3 \gamma+1) B^{3(\gamma+1)(\alpha+1)}}{\left[B^{3(\gamma+1)(\alpha+1)}-A R^{3(\gamma+1)(\alpha+1)}\right]^{\frac{\alpha}{\alpha+1}}}<0
$$

From the expression (7) and (11), when the cosmological radius tends to a finite value $R^{*}$, then $\dot{R}=0$, and $\ddot{R}=-\infty$, i.e., the declaration is infinite. Thus, the stop of expansion occurs in a singular way that means BigBrake Singularity.

Let us notice that the Big-Brake Singularity just like other soft singularities possesses an important property the Christoffell Symbols at the singularity are finite (or even zero). Thus the matter can pass through this singularity and then the geometry of the space-time can re-appear. It is easy to show that the expression (11) are well defined at $R>R^{*}$ namely, after arriving at the point of Big-Brake $R\left(t_{B}\right)=R^{*}$, which is at the same time the point of the maximal expansion of the universe, the universe begin contracting and this contraction culminates in the encounter with a Big-Crunch Singularity at $R=0$. Thus the model describes the evolution of the universe from the Big-Bang to the Big-Crunch passing through the soft Big-Brake singularity at the moment of the maximal expansion of the universe.

If we want to have little bit more rich model for the analysis of its classical and quantum dynamics, we can use rather a standard procedure of the re-construction of potentials of minimally coupled scalar fields, reproducing a given cosmological evolution. This procedure is based on use of two equations :

$$
\begin{aligned}
& \dot{\phi}=p+\rho \\
& V=\frac{1}{2}(\rho-p)
\end{aligned}
$$

the result of the reconstruction procedure for details see [A.Y. Kamenshchik, C. Keifer Phys. Rev D76, 064032 (2007)] 


$$
V(\phi)= \pm \frac{\sqrt{A}}{2}\left(\sinh 3 \phi-\frac{1}{\sinh 3 \phi}\right)
$$

As a matter of fact we have two possible potentials, which differs by the sign. The Big-Brake singularity occurs when the energy density $\rho=0$ and the pressure $p$ is positive and infinite. To achieve this condition in the scalar field model it is necessary to require that the potential is negative and infinite and this occurs from equation (14) when $\phi \rightarrow 0$ being positive. Thus to have the model with Big-Brake singularity we can consider the scalar field with the potential as

$$
V=-\frac{V_{0}}{\phi} \quad \text { [where } V_{0} \text { is positive constant] }
$$

The Klein-Gordon equation for the scalar field with the potential (15) is

We have from (2) and (12), (13),

$$
\ddot{\phi}+3 H \dot{\phi}+\frac{V_{0}}{\phi^{2}}=0
$$

$$
V=\frac{\dot{R}^{2}}{R^{2}}-\frac{\dot{\phi}^{2}}{2}, \text { and } H^{2}=V+\frac{\dot{\phi}^{2}}{2}
$$

Hence from (15) and (17), we get,

$$
H^{2}=\frac{\dot{\phi}^{2}}{2}-\frac{V_{0}}{\phi}
$$

which is the first Friedmann equation differentiating (18) with respect to the time $t$ and using (16), we get,

$$
\dot{H}=-\frac{3}{2} \dot{\phi}
$$

Now we shall construct the complete classification of the cosmological evolutions (trajectories) of our model.

\section{The quantum dynamics of the scalar field potentials:}

Using the equation (15) with canonical formalism and the Wheeler-DeWitt equation, the action of the Friedmann flat model with the minimally coupled scalar field of the metric (1) as

$$
S=\int d t\left(\frac{R^{3} \phi^{2}}{2 N}-R^{3} V(\phi)-\frac{R \dot{R}^{2}}{N}\right)
$$

We take $N=1$, and we define the canonical conjugated momenta as

$$
p_{\phi}=\frac{R^{3} \phi}{N}
$$

and, $\quad p_{R}=-\frac{R \dot{R}}{N}$

The Hamiltonian is

$$
H=N\left(-\frac{p_{R}^{2}}{4 R}+\frac{p_{\phi}^{2}}{2 R^{3}}+V R^{3}\right)
$$

The variation of the action with respect to $N$ gives

$$
-\frac{p_{R}^{2}}{4 R}+\frac{p_{\phi}^{2}}{2 R^{3}}+V R^{3}=0
$$

and the implementation of the Dirac quantization procedure gives the Wheeler De-Witt equation as

$$
\left(-\frac{\hat{p}_{R}^{2}}{4 R}+\frac{\hat{p}_{\phi}^{2}}{2 R^{3}}+V R^{3}\right) \psi(R, \phi)=0
$$


where, $\psi(R, \phi)$ is the wave function of the universe. Considering the momenta as $\hat{p}_{R}=-i \frac{\partial}{\partial R}, \hat{p}_{\phi}=-i \frac{\partial}{\partial \phi}$ the equation (25) becomes,

$$
\left(\frac{R^{2}}{4} \frac{\partial^{2}}{\partial R^{2}}-\frac{1}{2} \frac{\partial^{2}}{\partial \phi^{2}}-\frac{R^{6} V_{0}}{\phi}\right) \psi(R, \phi)=0 \text { [using equation (15)] }
$$

The interpretation of the wave function of the universe is rather an involved question mark. The point is that to choose the measure in the space of the corresponding Hilbert-Space we should fix a particular gauge condition, eliminating in such a way the redundant gauge degrees of freedom and introducing a temporal dynamics into the model. Assuming generally that the cosmological radius $R$ is in some way connected with the chosen time parameter and that the unique physical variable is the scalar field $\phi$. So the solution of the equation (26) may be in the form

$$
\psi(R, \phi)=\sum_{n=0}^{\infty} C_{n}(R) \chi_{n}(R, \phi)
$$

where $\chi_{n}$ satisfy the equation

$$
\left(-\frac{1}{2} \frac{\partial^{2}}{\partial \phi^{2}}-\frac{R^{6} V_{0}}{\phi}\right) \chi(R, \phi)=-E_{n}(R) \chi_{n}(R, \phi)
$$

while the functions $C_{n}(R)$ satisfy the equation

$$
\frac{R^{2}}{4} \frac{\partial^{2}}{\partial R^{2}} C_{n}(R)=E_{n}(R) C_{n}(R)
$$

where $n=0,1,2, \ldots$ requiring the normalizability of functions $\chi_{n}$ on the interval $0 \leq \phi<\infty$, which, in turn, implies their non-singular behavior at $\phi=0$ and $\phi \rightarrow \infty$ and using the considerations similar to those used in the analysis of the Schrödinger equation for the hydrogen-like atoms, one can show that the acceptable values of $E_{n}$ are

$$
E_{n}=\frac{V_{0} R^{12}}{2(n+1)^{2}}
$$

and the corresponding eigen functions are as,

$$
\chi_{n}(R, \phi)=\phi \exp \left(-\frac{V_{0} R^{6} \phi}{n+1}\right) L_{n}^{1}\left(\frac{2 V_{0} R^{6} \phi}{n+1}\right)
$$

where $L_{n}^{1}$ are the associated Laguerre polynomials.

The above equation (31) disappear at $\phi=0$, where the universe encounters the Big-Brake and also consider at the Big-Rip when the universe was in another phase, can be interpreted as the disappearance of the wave function of the universe and hence a quantum avoidance of the classical Big-Brake singularity and Big-Rip Singularity. We have also seen that the wave function of the universe disappear at $\phi \rightarrow \infty$. This value of the scalar field corresponds to both the Big-Bang and Big-Crunch Singularities. So in this two singularities the scalar-field tends to infinity and hence the potential term disappears and we remain only with the kinetic term. That means, in turn, that the scalar field becomes effectively mass-less and its energy-momentum tensor becomes equivalent to that of the stiff matter. In other words, the scalar field matter degenerates into the perfect fluid and the family of the trajectories degenerates into one trajectory in the neighbor-hood of the Big-Bang and the Big-Crunch singularities. Let us emphasize that it is true for our particular potential of the scalar-field. Other potentials, perhaps, can demonstrate other quantum and classical behaviors.

At the Big-Bang and Big-Crunch Singularities when the scalar field potential $V=0$, then we have from the Friedmann equation (17)

$$
\frac{\dot{R}^{2}}{R^{2}}=\frac{\dot{\phi}^{2}}{2} \text { solving this, we have } R=R^{*} e^{ \pm \frac{\phi}{\sqrt{2}}}
$$


Now we would like to study the case of negative values of the scalar field $\phi$. This case is equivalent to the equation (15) where $V_{0}$ is negative. The equations for the functions $\chi$ are similar to that for the charged particle in the repelling Coulomb potential. As is well known, it is impossible to have the eigenfunction, regular at both zero and infinite values of the scalar field, which in other words corresponds to the absence of bounded states for the repelling potentials. Nevertheless, one can construct the wave packets providing the regular behavior and the normalizability of the full wave function. In the cosmological case the explicit construction of such packets requires a definition of the gauge condition and the measure on the corresponding case. If we shall not consider this problem here. Instead, one can undertake some qualitative considerations. The eigen functions, used for the construction of the wave packets should disappear at the zero value of the scalar field. Instead, they cannot disappear at the infinity and should behave as a partial waves in the scattering theory. However, the wave packets should be constructed in such a way that the full wave function at the infinity should vanish due to the destructive interference. Thus, the probabilities should vanish at both for the zero value of the scalar-field.

\section{The Tachyon and Pseudo-tachyon Cosmological Model:}

The tachyon field is today considered as one of the possible candidates for the role of dark energy, which was born in the context of the string theory provides an example of matter having a large enough negative pressure to produce an acceleration of the expansion rate of the universe. The tachyon models represent a subclass of the models with non-standard kinetic terms, which descend from the Born-Infeld model. We first write down the general formulae of the tachyon cosmology.

The Lagrangian of the tachyon field $T$ is

$$
L=-\sqrt{-g} V(T) \sqrt{1-g^{\mu v} T,{ }_{\mu} T,_{v}}
$$

or for the spatially homogeneous tachyon field as,

$$
L=-\sqrt{-g} V(T) \sqrt{1-\dot{T}^{2}}
$$

where $g$ is the determinant of the metric. The energy density of this field is

$$
\rho=\frac{V(T)}{\sqrt{1-\dot{T}^{2}}}
$$

and the pressure, $p=-V(T) \sqrt{1-\dot{T}^{2}}$

The Klein-Gordon field equation is

$$
\frac{\ddot{T}}{1-\dot{T}^{2}}+3 H \dot{T}+\frac{V_{, T}}{V(T)}=0
$$

We shall introduce also the pseudo-tachyon field with respect to the space $R_{I}(=i R)$ with the Lagrangian, $L=\sqrt{-g} W(T) \sqrt{\dot{T}^{2}-1}$

with the energy density and pressure as

$$
\begin{aligned}
& \rho=\frac{W(T)}{\sqrt{\dot{T}^{2}-1}} \\
& p=W(T) \sqrt{\dot{T}^{2}-1}
\end{aligned}
$$

and the field equation as, $\frac{\ddot{T}}{1-\dot{T}^{2}}+3 H \dot{T}+\frac{W_{, T}}{W(T)}=0$

when $V(T)$ is a constant, the cosmological model coincide with the Chaplygin gas model (for $\gamma=0$ and $A<0$ ) and for $W(T)$ is a constant then the cosmological model coincide with the anti-Chaplygin gas model $(\gamma=0$ and $A>$ 0 ). Now, solving the Klein-Gordon equation (37) and (41) simultaneously for constant $V(T)$ and $W(T)$, we get,

$$
\frac{\dot{T}^{2}}{1-\dot{T}^{2}}=\frac{D^{2}}{R^{6}}
$$

[where $D^{2}$ is an integration constant]

For constant $V(T)$ and $W(T)$, we have from the equation (35) and (39),

$$
D^{2}=-R^{* 6}
$$

since at $R=R^{*}$, then $\rho=0$. Thus, we have from (42) and (43), 


$$
\dot{T}^{2}=\frac{1}{1-\frac{R^{6}}{R^{* 6}}}
$$

where $R \rightarrow R^{*}$, or $R_{I} \rightarrow R_{I}^{*}$, the $\dot{T} \rightarrow \pm \infty$, while $R \rightarrow 0$ then $\dot{T} \rightarrow \pm 1$ and $R \rightarrow \infty$ then $\dot{T} \rightarrow 0$. Thus for constant Tachyon or Pseudo-Tachyon field (i.e., $T=$ constant) for $R \rightarrow \infty$ i.e., at Big-Rip Singularity, but, at the Big-Bang or Big-Crunch Singularity the said field $T$ have time dependent (i.e., $T \rightarrow \pm t+C$, where ' $t$ ' is a cosmological time and $C$-being the integration constant).

Now we shall study a very particular tachyon potential depending on the trigonometrical functions which was suggested in the paper published by $V$. Gorini and others, Phys. Rev. D69. 123512 (2004) is the form

$$
V(T)=\frac{\Lambda}{\sin ^{2} \frac{3}{2} \sqrt{\Lambda(1+k) T}} \times \sqrt{1-(1+k) \cos ^{2} \frac{3}{2} \sqrt{\Lambda(1+k) T}},
$$

where $\Lambda$ is a positive constant and $-1<k<1$. In this case one subset of the cosmological evolutions are infinite and tend to a de-Sitter regime of the exponential expansion with the asymptotic value of the tachyon field $T=T_{0}=\frac{\pi}{3 \sqrt{\Lambda(1+k)}}$. Other trajectories go to the points of the two-dimensional phase space $(T, \dot{T})$, where the field acquires the values $\quad T=T_{3}=\frac{2}{3 \sqrt{\Lambda(1+k)}} \arccos \frac{1}{\sqrt{1+k}} \quad$ or $T=T_{4}=\frac{2}{3 \sqrt{\Lambda(1+k)}}\left(\pi-\arccos \frac{1}{\sqrt{1+k}}\right)$ and where the expression under the sign of the square root of the potential (45) vanishes and then $\dot{T}= \pm 1$, thus $L=0$, by the equation (34). It was shown in the above said paper that after the transformation of the tachyon into the pseudo-tachyon becomes unavoidable. The potential $W(T)$ is obtained from (45), by $W=i V$, where, $i=\sqrt{-1}$ What happens after that? Let us suppose that the trajectory of the tachyon field cross the point $T=T_{3}, \dot{T}=-1$. Then, after crossing this point the universe tends to the Big-Brake Singularity in the regime, which is described by,

$$
T=T_{B B}+\left(\frac{4}{3 W\left(T_{B B}\right)}\right)^{1 / 3}\left(t-t_{B B}\right)^{1 / 3}
$$

and,

$$
H=\left(\frac{9 W^{2}\left(T_{B B}\right)}{2}\right)^{1 / 3} \cdot\left(t-t_{B B}\right)^{1 / 3}
$$

where $t_{B B}$ and $T_{B B}$ are the time-moment and tachyon field at Big-Brake Singularity. It was shown that $0<T_{B B} \leq T_{3}$ and hence the cosmological trajectories encountering the Big-Brake singularity constitute an infinite one parameter set, whose elements can be parameterized by the value of the tachyon field $T_{B B}$. Then, after the Big-Brake crossing the cosmological expansion is followed by the contraction, which culminates at the encounter with the Big-Crunch singularity, which occurs at $T=0$ at constant and $\dot{T}=-\sqrt{\frac{1+k}{k}} \quad[$ where $-1<k$ $<1]$

Crossing the Big-Crunch singularity $k$ lies between 0 and -1 (i.e., $-1<k<0$ ), the potential energy changes to the kinetic energy after changing the phase of the universe and considering the radius of the universe such as $R_{I}^{2}\left(=-R^{2}\right.$ ) (the pseudo-space $\left.R_{I}=i R\right)$. The universe is then going to the Big-Rip singularity.

\section{Cosmological Explanation by the Gaussian Energy Group:}

After crossing the Big-Crunch Singularity the energy can be interpreted by the Gaussian unitary energy group SU(6), a new type of energy group, called, latent energy group. We consider a special unitary group of degree $\mathrm{n}$ as $\mathrm{SU}(n) \subset \mathrm{U}(\mathrm{n})$, where $\mathrm{U}(n)$ is a $\mathrm{n} \times \mathrm{n}$ unitary matrices with determinant 1 , which is a sub-set of the general linear group $\operatorname{GL}(n, C)$, where ' $C$ ' is a classical lie group. 
Now, $\mathrm{SU}(n) \supset \mathrm{SU}(p) \times \mathrm{SU}(n-p) \times \mathrm{U}(1)$

putting $p=5, n=11$, where $p>1$ and $n-p>1$.

So that $\mathrm{SU}(11) \supset \mathrm{SU}(5) \times \mathrm{SU}(6) \times \mathrm{U}(1)$

For completeness there also the orthogonal and sympletic subgroups $\mathrm{SU}(11) \supset \mathrm{O}(11)$; $\mathrm{SU}(22) \supset \mathrm{US}_{p}(22)$ since the rank of SU(11) is 10 and U(1) is 1, a useful check is that the sum of the ranks of the subgroup SU(5) and $\mathrm{SU}(6)$ is less than or equal to the rank of the original group. From Big-Crunch to Big-Rip singularity, the time derivative of the pseudo-potential field $\dot{T}=-\sqrt{\frac{1+k}{k}}$ exists in the range $-1<k<0$, we consider then the Gaussian energy group $\mathrm{SU}(5)$ changes to the energy groups $\mathrm{SU}(6)$, a new type of energy sources other than $\mathrm{SU}(5) \supset \mathrm{SU}(3) \times \mathrm{SU}(2) \times \mathrm{U}(1)$.

\section{Hamiltonian formalism for the tachyon and pseudo-tachyon field:}

Using the metric (1), one can see that the contribution of the tachyon field into the action is,

$$
s=-\int d t N R^{3} V(T) \sqrt{1-\frac{\dot{T}^{2}}{N^{2}}}
$$

The conjugate momentum for $T$ is

$$
p_{T}=\frac{R^{3} V \dot{T}}{N \sqrt{1-\frac{\dot{T}^{2}}{N^{2}}}}
$$

Also,

$$
\dot{T}=\frac{N p_{T}}{\sqrt{p_{T}^{2}+R^{6} V^{2}}}
$$

The Hamiltonian of the tachyon field is

$$
H=N \sqrt{p_{T}^{2}+R^{6} V^{2}}
$$

Analogously for the pseudo-tachyon field

$$
\begin{aligned}
& p_{T}=\frac{R^{3} W \dot{T}}{N \sqrt{\frac{\dot{T}^{2}}{N^{2}}-1}} \\
& \dot{T}=\frac{N p_{T}}{\sqrt{p_{T}^{2}-R^{6} W^{2}}} \\
& H=N \sqrt{p_{T}^{2}-R^{6} W^{2}}
\end{aligned}
$$

Considering $N=1$ and adding the gravitational part of the Hamiltonian and quantizing the corresponding observables we obtain the following Wheeler-DeWitt equations for the tachyons $\left(\sqrt{\hat{p}_{T}^{2}+R^{6} V^{2}}-\frac{R^{2} \hat{p}_{a}^{2}}{4}\right) \psi$ $(R, T)=0$

and for pseudo tachyons $\left(\sqrt{\hat{p}_{T}^{2}-R^{6} W^{2}}-\frac{R^{2} \hat{p}_{a}^{2}}{4}\right) \Psi(R, T)=0$

The Hamiltonian depends non-polynomially on the conjugate momentum of the universe filled with tachyon or pseudo-tachyon field. Hence, the study of the above wheeler-DeWitt equations (58) and (59) is very difficult. However, one can come to some qualitative conclusions, considering some particular models. The Hamiltonian in the equation (59) does not depend on the field $T$. Thus, it is more convenient to use the representation of the quantum state of the universe by the canonical change of $\hat{p}_{a} \equiv-i \frac{\partial}{\partial R}$, where it depends on the co-ordinate $R$ and the momentum $p_{T}$. Then the equation (59) becomes, 


$$
\left(\sqrt{p_{T}^{2}-R^{6} W^{2}}+\frac{R^{2}}{4} \frac{\partial^{2}}{\partial R^{2}}\right) \psi\left(R, p_{T}\right)=0
$$

The Hamiltonian of the equation (60) is well defined at $p_{T}^{2} \geq R^{6} W^{2}$.

When $p_{T}^{2} \rightarrow R^{6} W^{2}$, then from equation (56), we have $\dot{T}^{2} \rightarrow \infty$, which, in turn, corresponds to the encounter with the Big-Brake singularity. Again if we consider $p_{T}^{2} \leq R^{6} W^{2}$, then it is require that the wave function of the universe

$$
\psi\left(R, p_{T}\right)=0
$$

But if we consider there exist pseudo-space $R_{I}(=i R)$ then equation (60) becomes,

$$
\left(\sqrt{p_{T}^{2}+R_{I}^{6} W^{2}}+\frac{R_{I}^{2}}{4} \frac{\partial^{2}}{\partial R_{I}^{2}}\right) \psi\left(R_{I}, p_{T}\right)=0
$$

which shows the Hamiltonian is well defined in the pseudo-tachyon field in respect to the pseudo-space $R_{I}$.

The condition (61) is equivalent to the quantum avoidance of the Big-Brake singularity. Again, it was noticed from the equation (44), at Big-Bang or Big-Crunch singularity $\dot{T}^{2}=1$. Hence from equation (55), $\left|p_{T}\right| \rightarrow \infty$. A general requirement of the normalizability of the wave function of the universe implies the vanishing of $\psi\left(R, p_{T}\right)$ at $p_{T} \rightarrow \pm \infty$ which signifies the quantum avoidance of the Big-Bang and BigCrunch singularities. Such a quantum behavior has its classical counterpart. Thus, we have seen from the equation (62), one can always choose such a quantum state, which does not disappear at any value of $R_{I}$ which guarantees the positivity of the quantum average of the operator. Coming back to our cosmological model we can say that the requirements of the well-definiteness of the Pseudo-Tachyon part of the Hamiltonian operator in the Wheeler-DeWitt equation, does not imply the disappearance of the wave function of the universe at some values of the variables and thus, does not reveal the effect the quantum avoidance of the cosmological singularity. Let us notice that this is the only case with the absence of the effect of avoidance and it has its classical counterpart. Indeed, we have seen in the preceding section that there was a one-parameter family of cosmological evolutions encountering the Big-Brake and Big-Rip singularities. In the scalar field model, the potential is inversely proportional to the field itself, hence a cosmological evolution, including Big-Rip and crossing the Big-Brake singularity is not a unique one, but belongs to a large family.

Thus we conclude that the universe having two phase: one starting from Big-Bang to Big-Crunch through Big-Brake singularity and another starting from Big-Crunch to Big-Bang through Big-Rip singularity, where the radius of the universe being infinity and also the rate of expansion is infinity having pressure and density depending on some parameters $\gamma, A$, $\alpha$ of the equation (8) and (9), if we consider $\gamma=-1$, then the energy pressure and density will be infinity, but for $A=0, \gamma \neq-1$ then density will be zero. Again, from equation (55), (56) and (57) and (48) substituting $R_{I}=i R$, we have in the other phase of the universe,

$$
\begin{aligned}
& p_{T}=V \sqrt{1+k} R_{I}^{3} \text { [putting } W=i V \& N=1 \text { ] } \\
& \text { and, } \frac{p_{T}}{\sqrt{p_{T}^{2}+R_{I}^{2} W^{2}}}=\dot{T}=-\sqrt{\frac{1+k}{k}} \\
& \text { and, } H=\sqrt{p_{T}^{2}+R_{I}^{2} W^{2}}
\end{aligned}
$$

Thus, from (65), we conclude, always $H>0$, so there is an eternal universe for $R_{I} \rightarrow \infty$. Again from the equation (54), the Hamiltonian of the Tachyon field always positive, thus there exists a full wave. For $p_{T} \rightarrow \infty$, then $H \rightarrow \infty$. If we compare the equation (6) with (35) or (39), we have,

$$
V(T)=\frac{\sqrt{1-\dot{T}^{2}}}{(\gamma+1)^{1 /(\alpha+1)}}\left[\left(\frac{B}{R}\right)^{3(\gamma+1)(\alpha+1)}-A\right]^{\frac{1}{\alpha+1}}
$$


and,

$$
W(T)=\frac{\sqrt{\dot{T}^{2}-1}}{(\gamma+1)^{1 /(\alpha+1)}}\left[\left(\frac{B}{R}\right)^{3(\gamma+1)(\alpha+1)}-A\right]^{\frac{1}{\alpha+1}}
$$

Also, we have from equation (2) and (36) and (40),

and,

$$
\begin{aligned}
& V(T)=-\frac{\gamma \rho+\frac{A}{\rho^{\alpha}}}{\sqrt{1-\dot{T}^{2}}} \\
& W(T)=-\frac{\gamma \rho+\frac{A}{\rho^{\alpha}}}{\sqrt{\dot{T}^{2}-1}}
\end{aligned}
$$

Thus at Big-Rip singularity, when $R \rightarrow \infty$, i.e., $R_{I} \rightarrow \infty$, then, we have from the equation (8) and (35) or (39),

$$
\begin{aligned}
& V(T)=\frac{\sqrt{1-\dot{T}^{2}}[-A]^{\frac{1}{1+\alpha}}}{(\gamma+1)^{\frac{1}{(\alpha+1)}}} \\
& W(T)=\frac{\sqrt{\dot{T}^{2}-1}[-A]^{\frac{1}{1+\alpha}}}{(\gamma+1)^{\frac{1}{(\alpha+1)}}}
\end{aligned}
$$

and also from equation (9) and (36) or (40) we get,

and,

$$
V(T)=-\frac{A}{\sqrt{1-\dot{T}^{2}}} \frac{1}{[-A]^{\alpha /(\alpha+1)} \cdot(\gamma+1)^{1 /(\alpha+1)}}
$$

Now, consider at Big-Crunch singularity, $\dot{T}=-\sqrt{\frac{1+k}{k}},[-1<k<1]$ putting the value of $\dot{T}$ in the equation from (66) to (73), we have, $1-\dot{T}^{2}=1-\left(\frac{1}{k}+1\right)=-\frac{1}{k}$

$$
\text { or, } \quad \dot{T}^{2}-1=\frac{1}{k}
$$

From (74) it is clear for real value of $\sqrt{1-\dot{T}^{2}}$, then $-1<k<0$ but for $\sqrt{\dot{T}^{2}-1}$, then $0<k<1$. From (66) and (74),

$$
V(T)=\frac{1}{\sqrt{-k}(\gamma+1)^{1 /(\alpha+1)}}\left[\left(\frac{B}{R}\right)^{3(\gamma+1)(\alpha+1)}-A\right]^{1 /(\alpha+1)}
$$

From (67) and (75),

$$
W(T)=\frac{1}{\sqrt{k}(\gamma+1)^{1 /(\alpha+1)}}\left[\left(\frac{B}{R}\right)^{3(\gamma+1)(\alpha+1)}-A\right]^{1 /(\alpha+1)}
$$

From (68) and (74), 


$$
V(T)=-\frac{1}{\sqrt{-k}}\left(\gamma \rho+\frac{A}{\rho^{\alpha}}\right)
$$

From (69) and (75),

$$
W(T)=\frac{1}{\sqrt{k}}\left(\gamma \rho+\frac{A}{\rho^{\alpha}}\right)
$$

From (70) and (74),

$$
V(T)=\frac{[-A]^{\frac{1}{1+\alpha}}}{\sqrt{-k}(\gamma+1)^{1 /(\alpha+1)}}
$$

From (71) and (75),

$$
W(T)=\frac{[-A]^{\frac{1}{1+\alpha}}}{\sqrt{k}(\gamma+1)^{1 /(\alpha+1)}}
$$

From (72) and (74),

From (73) and (75),

$$
V(T)=-\frac{A}{\sqrt{-k}} \frac{1}{[-A]^{\frac{\alpha}{1+\alpha}}(\gamma+1)^{1 /(\alpha+1)}}
$$

$$
W(T)=\frac{A}{\sqrt{k}} \frac{1}{[-A]^{\frac{\alpha}{1+\alpha}}(\gamma+1)^{1 /(\alpha+1)}}
$$

[From equation (76) to (83), where $-1<k<1$ ]

If we interested for the positive value of $k$, then one subset of the cosmological evolutions are infinite and tend to a de-Sitter regime of the exponential expansion with the asymptotic value of the tachyon field $T=$ $T_{0}=\frac{\pi}{3 \sqrt{\Lambda(1+k)}}$, where $\Lambda$ is a positive constant. But if we interested with the negative value of $k$, then what happen?

$$
1-\dot{T}^{2}>0 \text {, or, } \dot{T}^{2}<1 \text {. }
$$

The generalized Chaplygin gas model is described by a perfect fluid obeying an exotic equation of state $p=-\frac{A}{\rho^{\alpha}}$, where $0<\alpha \leq 1$, i.e., in the equation (2), where $\gamma=0$ and $A<0$. The standard Chaplygin gas correspond to $\alpha=1$. As well will see, using appropriate thermodynamics descriptions, the Chaplygin gas in the early times represents a dust matter behavior and mimics the positive cosmological constant at the late time. This property allows us to have a negative cosmological constant in the early universe in-spite of the fact that observations agree with the positive cosmological constant in the present epoch.

If we are motivated by the brane world scenarios where the matter is to be confined to the 4dimensioinal universe, i.e., auxiliary hyper-space, so all the components of the energy-momentum tensor $T_{A B}$ is set to zero but the space-time components and it means no matter escapes through the extra-dimension. We assume the energy-momentum tensor $T_{\mu \nu}$ to be that of an exotic $\chi$ fluid with the equation of state $p_{\chi}=\gamma \rho_{\chi}$, where $-1 \leq \gamma \leq-\frac{1}{3}$, so that there is violation of strong energy condition and the universe experiences accelerated expansion, it may be compared with equation (2), where $A=0$, from (79), $W(T)=\frac{\gamma_{0}}{\sqrt{K}} \rho$

VII. Classical dynamics of the universe with a scalar-field model:

We shall construct the complete classification of the cosmological evolutions (trajectories) of our model, using equation (16), (18) and (19). Let us consider the possible trajectories which cross the Big-Brake 
singularity. It was already noticed that such a singularity can be found when $\phi \rightarrow 0$, being positive. We consider the field $\phi$ approaching the Big-Brake singularity in the form,

$$
\phi(t)=\phi_{1}\left(t_{B}-t\right)^{\beta}
$$

Where $\phi$ and $\beta$ are positive constants and $t_{B}$, the moment of Big-Brake singularity. Substituting the above expression in the equation (18) and putting $H=0$, we obtain

$$
\frac{1}{2} \beta^{2} \phi_{1}^{2}\left(t_{B}-t\right)^{2 \beta-2}=\frac{V_{0}}{\phi_{1}}\left(t_{B}-t\right)^{-\beta}
$$

Comparing the power of $\left(t_{B}-t\right)$ of both sides of the above expression, we get,

$$
2 \beta-2=-\beta \text { i.e. } \quad \beta=\frac{2}{3}
$$

and,

$$
\frac{1}{2} \beta^{2} \phi_{1}^{2}=\frac{V_{0}}{\phi_{1}} \text {, i.e., } \phi_{1}=\left(\frac{9 V_{0}}{2}\right)^{1 / 3}
$$

Thus, $\quad \phi(t)=\left(\frac{9 V_{0}}{2}\right)^{1 / 3}\left(t_{B}-t\right)^{2 / 3}$

Substituting the above expression of $\phi(t)$ in the equation (19) and then integrating, we get,

$$
H=2\left(\frac{9 V_{0}}{2}\right)^{2 / 3}\left(t_{B}-t\right)^{1 / 3}+H_{B}
$$

[where $H_{B}$ is the integration constant]

In the above expression $H_{B}=0$ or, $H_{B} \neq 0$, for the moment of the maximal expansion of the universe coincides with that of the soft singularity crossing and the universe encounters the Big-Brake singularity. For $H_{B}>0$ or $H_{B}<0$, i.e., the sign of $H_{B}$ can be both positive or negative, hence, the universe can pass through this singularity in the phase of its expansion or of its contraction and where the energy density of the matter in the universe is different from zero. Again from the equation (90), it was noticed at the moment when $\phi=0$, shows that after passing the zero value, the scalar-field is positive it cannot change the sign. Now, we have differentiate both sides with respect to ' $t$ ' of the equation (90), we get,

$$
\dot{\phi}(t)=-\frac{2}{3}\left(\frac{9 V_{0}}{2}\right)^{1 / 3}\left(t_{B}-t\right)^{-1 / 3}
$$

which shows that $\dot{\phi}(t)$ is negative before crossing of the soft singularity and after its crossing it is positive. But it cannot change the sign in a singular way because the condition $\dot{\phi}\left(t_{0}\right)=0, \phi\left(t_{0}\right) \neq 0$ are incompatible with Friedmann equation (18).

So, the impossibility of the changing the sign of $\dot{\phi}(t)$ without the soft singularity crossing implies the inevitability of the approaching of the universe to this soft singularity. Also we have from equation (19) that the value of the Hubble parameter is decreasing during all the evolution.

And, $\quad|\dot{H}| \propto \dot{\phi}^{2}$

which shows that the absolute value of its time derivative is growing after the soft singularity crossing. That means that at some moments the Hubble parameter should change its sign, becoming negative. The change of the sign of the Hubble parameter is nothing but the passing through the point of the maximal expansion of the universe, after which it begins contraction culminating in the encounter with the Big-crunch singularity.

Thus all the cosmological evolutions where the scalar field has positive values have the following structure. They begin in the big-bang singularity with an infinite positive value of the scalar-field, then they pass through the soft singularity where the value of the scalar field is zero. All the trajectories also pass through the point of the maximal expansion and this passage through the point of the maximal expansion can precede or follow the passage through the soft singularity. In the case when these two moments coincide $\left(H_{B}=0\right)$, we have the Big-Brake singularity.

Now, both the terms in the right-hand-side of the equation (18), potential and kinetic, are positive and, hence, the Hubble parameter cannot disappear or change its sign. It can only tend to zero asymptotically while 
both these terms tend asymptotically to zero. Thus, in this case there are two possible regimes. An infinite expansion which begins with the Big-Bang singularity and an infinite contraction which culminates in the encounter with Big-Crunch singularity. The second regime can be obtained by the time reversal of the first one and vice-versa. Let us consider the expansion regime. We cannot consider

$$
\phi(t)=-\phi_{1}\left(t_{0}-t\right)^{\beta}
$$

where $\phi_{1}<0 ; \beta>0$, because it is incompatible with the equations (18) and (19). Hence, the potential term is always non-singular, and at the birth of the universe from the Big-Bang singularity the kinetics term dominates and the dynamics is that of the theory with the mass-less scalar field, namely,

$$
\phi(t)=\phi_{0}+\sqrt{\frac{2}{9}} \log t, H(t)=\frac{1}{3 t}
$$

[where $\phi_{0}$ is a constant]

Similarly after the death of the universe from the Big-Crunch singularity, the Kinetic terms were again much more active and hence the mass-less particles were moving randomly in arbitrary direction in another phase.

Consider the FRW space-time, as

\section{Massive Scalar field with complex scale factor:}

$$
d S^{2}=d t^{2}-a^{2}(t) d x^{2} .
$$

Here we consider ' $a$ ' as a complex scale factor, i.e., a $=R+i R_{I}$, where $a$ stands in the massive scalar-field $\phi$ has the action

$$
S=\int d t L=\int d t \int d^{3} x \sqrt{-g} \frac{1}{2}\left(g^{\mu v} \partial_{\mu} \phi \partial_{\nu} \phi-m^{2} \phi^{2}\right)
$$

In the Hamiltonian formalism the action with II $=a^{3} \dot{\phi}$

$$
\text { i.e., } \quad S=\int d t(\dot{\phi} \Pi-H)
$$

is given by the Hamiltonian and its density,

$$
\begin{aligned}
& H(t)=\int d^{3} x\left[\frac{\Pi^{2}}{2 a^{3}}+\frac{1}{2}\left(\frac{(\nabla \phi)^{2}}{a^{2}}+m^{2}\right) \phi^{2}\right]=\int d^{3} x H(t, x) \\
& \text { i.e., } \quad H(t, x)=\frac{\Pi^{2}}{2 a^{3}}+\frac{1}{2}\left[\frac{(\nabla \phi)^{2}}{a^{2}}+m^{2}\right] \phi
\end{aligned}
$$

we decompose the above, we have, the field

$$
\phi(t, x)=\int \frac{d^{3} k}{(2 \pi)^{3 / 2}} \phi_{k}(t) e^{i k \cdot x}
$$

and conjugate momentum as, $\Pi(t, x)=\int \frac{d^{3} k}{(2 \pi)^{3 / 2}} \pi(t) e^{i k . x}$

then the Hamiltonian is an infinite sum of time-dependent oscillators

$$
H(t)=\int d^{3} k \frac{1}{2}\left(\frac{\dot{\pi}_{k}^{2}}{a^{3}}+a^{3} \omega_{k}^{2} \phi_{k}^{2}\right)=\int d^{3} k H_{k}(t)
$$

where, $\pi_{k}=a^{3} \dot{\phi}_{k}, \omega_{k}^{2}(t)=\frac{k^{2}}{a^{2}}+m^{2}$

and the Hamiltonian (103) has the meaning of $H_{k}(t)=H_{k}\left(\pi_{k}, \phi_{k}, \Sigma_{t}\right)$ on each space like hyper-surface $\Sigma_{t}$ and the Hamiltonian formalism describe the evolution from one hyper-surface $\Sigma_{t_{0}}$ to another $\Sigma_{t}$.

Again, the stress-energy-momentum tensor

$$
T_{\mu v}=\frac{2}{\sqrt{-g}} \frac{\delta S}{\delta g^{\mu \nu}}=\partial_{\mu} \Phi \partial_{v}-\frac{1}{2} g_{\mu \rho}\left(g^{\rho v} \partial_{\rho} \Phi \partial_{\sigma} \Phi-m^{2} \Phi\right)
$$


has also the Hamiltonian expression

$$
\begin{aligned}
& T_{00}=\frac{H}{a^{3}}, T_{0 i}=\frac{\Pi}{a^{3}} \partial_{i} \Phi \\
& T_{i j}=\frac{a^{2}}{2} \delta_{i j}\left(\frac{\Pi^{2}}{a^{6}}-m^{2} \Phi^{2}\right)+\left(\partial_{i} \phi \partial_{j} \phi-\frac{1}{2} \delta_{i j}(\nabla \Phi)^{2}\right)
\end{aligned}
$$

The stress-energy momentum tensor (107) can be decompose into Fourier modes as

$$
\begin{aligned}
& \phi_{k}^{2}:=\phi_{k} \phi_{-k}=\left(\frac{\phi_{k}+\phi_{-k}}{2}\right)^{2}+\left(\frac{\phi_{k}-\phi_{-k}}{2 i}\right)^{2} \\
& \pi_{k}^{2}:=\pi_{k} \pi_{-k}=\left(\frac{\pi_{k}+\pi_{-k}}{2}\right)^{2}+\left(\frac{\pi_{k}-\pi_{-k}}{2 i}\right)^{2}
\end{aligned}
$$

Thus $k$-denotes both the cosine and sin mode. Hence, the Hamiltonian equation for $H_{k}$ is the Fourier mode of the field equation as,

$$
\ddot{\phi}_{k}+3 \frac{\dot{a}}{a} \dot{\phi}_{k}+W_{k}^{2}(t) \phi_{k}=0
$$

Putting $a=R+i R_{I}$, then from (110), we get,

$$
\ddot{\phi}_{k}+3 \cdot \frac{\dot{R}+i \dot{R}_{I}}{R+i R_{I}} \dot{\phi}_{k}+\left(\frac{k^{2}}{\left(R+i R_{I}\right)^{2}}+m^{2}\right) \phi_{k}=0
$$

Separating real and imaginary parts we have,

$$
\begin{aligned}
& \ddot{\phi}_{k}+3 \cdot \frac{R \dot{R}-R_{I} \dot{R}_{I}}{R^{2}-R_{I}^{2}} \dot{\phi}_{k}+\left\{\frac{k^{2}}{R^{2}-R_{I}^{2}}+m^{2}\right\} \phi_{k}=0 \\
& \text { and } \quad \ddot{\phi}_{k}+\frac{3}{2}\left(\frac{\dot{R}_{I}}{R_{I}}+\frac{\dot{R}}{R}\right) \dot{\phi}_{k}+m^{2} \phi_{k}=0
\end{aligned}
$$

Let us assume, that, $H_{I}=\frac{\dot{R}_{I}}{R_{I}}$ and $H_{R}=\frac{\dot{R}}{R}$, the Hubble parameters respectively in both phases of the universe, then,

$$
\ddot{\phi}_{k}+\frac{3}{2}\left(H_{I}+H_{R}\right) \dot{\phi}_{k}+m^{2} \phi_{k}=0
$$

is a simple equation of $\phi_{K}$, solving this, we get,

$$
\phi_{k}(t)=e^{\frac{-3 H t}{4}}\left[A e^{H \sqrt{\frac{9}{16}-\frac{m^{2}}{H^{2}}} t}+B e^{-H \sqrt{\frac{9}{16}-\frac{m^{2}}{H^{2}}} t}\right]
$$

[where $\left.H=H_{I}+H_{R}\right]$.

\section{IX. dS-radiation in the planar coordinates.}

In the planar coordinates, $a(t)=e^{H t}$, where $H=H_{I}+H_{R} ; d S^{2}=-d t^{2}+e^{2 H t} d x^{2}$

then the general solution to the equation (110), in the metric (115) is given by

$$
\phi_{k}(t)=\left(\frac{\pi}{4 H}\right)^{1 / 2} e^{-3 H t / 2}\left[C_{k}^{(1)}-e^{-\pi p / 2} H_{i p}^{(1)}(z)+C_{k}^{(2)} e^{\pi p} H_{i p}^{(2)}(z)\right]
$$

where $H_{i p}^{(1)}$ and $H_{i p}^{(2)}$ are the Hankel functions and for massive particles $(m / H>3 / 2)$ 


$$
z=\frac{K}{H} e^{-H t}, p=\left(\frac{m^{2}}{H^{2}}-\frac{9}{4}\right)^{1 / 2}
$$

The equation (116) can be compared with the equation (114).

The standard quantization rule requires that the Wronskian condition should be satisfied,

$$
a^{3}(t) W_{r}\left[\phi_{k}(t) \cdot \phi_{k}^{*}(t)\right]=a^{3}(t)\left(\phi_{k}(t) \dot{\phi}_{k}^{*}(t)-\dot{\phi}_{k}(t) \phi_{k}^{*}(t)\right)=i 1
$$

Through the integration constants $C_{k}^{(1)}$ and $C_{k}^{(2)}$ can be arbitrary complex numbers, the quantization rule (118) restricts then to satisfy

$$
\left|C_{k}^{(1)}\right|^{2}-\left|C_{k}^{(2)}\right|^{2}=1
$$

Again we have from (104),

$$
H_{k}(t)=\frac{1}{2}\left(\frac{\dot{\pi}_{k}^{2}}{a^{3}}+a^{3} \omega_{k}^{2} \phi_{k}^{2}\right)
$$

where, $\pi_{k}=a^{3} \dot{\phi}_{k}, \omega_{k}^{2}=\frac{k^{2}}{a^{2}}+m^{2}$

i.e., $\quad H_{k}(t)=\frac{1}{2}\left\{a^{3} \ddot{\phi}_{k}^{2}+9 a \dot{a}^{2} \dot{\phi}_{k}^{2}+6 a^{2} \dot{a} \dot{\phi} \ddot{\phi}+a k^{2} \phi_{k}^{2}+a^{3} m^{2} \phi_{k}^{2}\right\}$

Again from (111), we have,

$$
\frac{\partial \phi}{\partial t}=\left[\frac{3}{2} \frac{R_{I} \dot{R}_{I}-R \dot{R}}{R^{2}-R_{I}^{2}} \pm \sqrt{\frac{9}{4}\left(\frac{R \dot{R}-R_{I} \dot{R}_{I}}{R^{2}-R_{I}^{2}}\right)-\frac{k^{2}}{R^{2}-R_{I}^{2}}-m^{2}}\right] \phi_{k}
$$

Integrating we get,

$$
\phi=A e^{\frac{3}{4} \log \left(R^{2}-R_{I}^{2}\right) \pm \int \sqrt{\frac{9}{4}\left(\frac{R \dot{R}-R_{I} \dot{R}_{I}}{R^{2}-R_{I}^{2}}\right)^{2}-\frac{K^{2}}{R^{2}-R_{I}^{2}}-m^{2}}} \delta t
$$

when $R$-increase and $R_{I}$ decrease, then $\phi$ decreases, i.e., the potential of the scalar field decreases as the universe expands, i.e., the potential energy of the scalar field are responsible for expanding the universe as dynamic.

\section{$X$. Gaussian wave functions:}

The time-dependent Hamiltonian (103) in an expanding universe may give an adverse feeling for the Schrödinger picture

$$
\left.i \frac{\partial}{\partial t} \Psi_{k}\left(\phi_{k}, t\right)=\hat{H}_{k}, t\right)=\hat{H}_{k}(t) \Psi_{k}\left(\phi_{k}, t\right)
$$

Also the Schrödinger picture indicates, quantum states are C-number wave functions that carry the same information as the Heisenberg field operator.

However, it has been known for a long time that the Gaussian wave function could be expressed in terms of a classical solution to the equation (110). It may be understood from the fact that the Heisenberg field operator also satisfied the same equation (110) and the Heisenberg picture provides the same quantum information as the Schrödinger picture. In fact, the Gaussian wave function on a space-like hyper-surface $\Sigma_{t}$ of constant $t$ for each Fourier mode, which is the solution to the Schrödinger (122) and is normalized to unity, is given by

$$
\psi_{k}\left(\phi_{k}, t\right)=\left(\frac{\phi_{k}}{\sqrt{2 \pi}\left|\phi_{k}\right|^{2}}\right)^{1 / 2} \exp \left(\frac{i a^{3}}{2} \frac{\dot{\phi}_{k}^{*}}{\phi_{k}^{*}} \phi_{k}^{2}\right)
$$

where $\phi_{K}$ is a complex solution to equation (110) that satisfies the quantization rule (118). The Hamiltonian (103) has the algebra SU(1,1) etc., for which we may choose a time-independent Hermitian basis.

\section{Hamiltonian formalism for the tachyon and pseudo-tachyon field:}

Using the metric, $d S^{2}=d t^{2}-a^{2} d x^{2}$ [Taking $\left.N=1\right]$

The Hamiltonian of the tachyon and pseudo tachyon field as 
$H=\sqrt{p_{T}^{2}+R^{6} V^{2}}$ [where $V$ and $R$ respectively potentials radius of the universe]

and, $\quad H=\sqrt{p_{T}^{2}+R_{I}^{6} W^{2}}$ [where $R_{I}=i R$ and $\left.W=i V\right]$

It may be compared with the Hamiltonian equation (103) with (120) we have,

$$
\begin{gathered}
\frac{1}{2} \int\left[R^{3} \ddot{\phi}_{k}+9 R \dot{R}^{2} \dot{\phi}_{k}^{2}+6 R^{2} \dot{R} \dot{\phi}_{k} \ddot{\phi}_{k}+R K^{2} \phi_{k}^{2}+R^{3} m^{2} \phi_{k}^{2}\right] d^{3} k \\
=\sqrt{p_{T}^{2}+R^{6} V^{2}}
\end{gathered}
$$

and,

$$
\begin{aligned}
& \frac{1}{2} \int\left[i R^{3} \ddot{\phi}_{k}+9 i R_{I} \dot{R}_{I}^{2} \dot{\phi}_{k}^{2}+6 i R_{I}^{2} \dot{R}_{I} \dot{\phi}_{k} \ddot{\phi}_{k}-i R_{I} K^{2} \phi_{k}^{2}+i R_{I}^{3} m^{2} \phi_{k}^{2}\right] d^{3} k \\
& =\sqrt{p_{T}^{2}+R_{I}^{6} W^{2}} \\
& \text { i.e., } \quad \frac{i}{2} \int\left[R_{I}^{3} \ddot{\phi}_{k}+9 R_{I} \dot{R}_{I}^{2} \dot{\phi}_{k}^{2}+6 R_{I}^{2} \dot{R}_{I} \dot{\phi}_{k} \ddot{\phi}_{k}-R_{I} K^{2} \phi_{k}^{2}+R_{I}^{3} m^{2} \phi_{k}^{2}\right] d^{3} k \\
& =\sqrt{p_{T}^{2}+R_{I}^{6} W^{2}}
\end{aligned}
$$

\section{Concluding Remark}

We have studied a relation between the cosmological singularities in classical and quantum theory, comparing the classical and quantum dynamics in three models possessing four type of singularities namely, Big-Bang, Big-Brake, Big-Crunch, and Big-Rip singularity. In this paper, we consider the universe having two types of space-time, one starting from Big-Bang to Big-Crunch through the Big-brake singularity (so called real universe) and other from Big-Crunch to Big-Bang through Big-Rip singularity, the space in this regime called Pseudo-Space. The above three models based on a scalar field, tachyon and pseudo-tachyon field model. In the pseudo-tachyon field, the Wheeler-DeWitt equation can be constructed, where it was required that the Hamiltonian operator are well defined. For this we assume a pseudo-space $R_{I}(=i R$, where ' $R$ ' is the radius of our unfolded universe), which ensure, that there exist a part of our universe with respect to the unfolded universe after Big-Crunch singularity to Big-Bang through Big-Rip singularity, where the radius of the universe being infinite towards Big-Rip and velocity of the mass-less scalar-field particle being infinite, having a negative pressure depending on some arbitrary constant parameters. There are some kind of classical-quantum correspondence, as it was shown in the classical tachyon and pseudo-tachyon model exists an infinite one parameter set of the cosmological evolutions. So the evolutions of our universe begins from PBH through BigBang singularity and all the massive stars formed by some black-holes possess up-to the Big-Brake singularity and also ending by the process of collapsing stars within a black-hole and hence ultimately the evolution of our universe ending through post mature black-hole through Big-Crunch singularity.

Also in my paper we have studied the production of massive particles in a di-Sitter space with complex scale factor $R+i R_{I}$, using the quantum dynamics that unified the invariant picture. In the quantum dynamics a massive field in an expanding FRW, universe or a $d S$-space is equivalent to an infinite sum of time-dependent oscillators in which the time-dependent-mass comes from the expanding spatial volume and the frequencies from the red-shifted or blue-shifted wave lengths. The main advantage of the invariant operator picture is that there exists the time-dependent annihilation and creation operators which generate the exact quantum states, the Gaussian wave function being the simplest one among them.

\section{Acknowledgement:}

The author is thankful to Prof. Subenoy Chakraborty, Department of Mathematics, Jadavpur University, Kolkata, West Bengal, India, for his helpful discussions.

\section{References:}

[1]. N.K. Bhadra, IOSRJM, H0244145, The Complex Model of the Universe, Issue 4, 41 (2012).

[2]. N.K. Bhadra, IOSRJM, D0412033, The Complex Model of the Quantum Universe, vol.4, Issue 1, 20 (2012).

[3]. A.Y. Kamenshchik and Serena-Manti, Phys Rev D85, 123518 (2012).

[4]. C.W. Misner, Phys Rev Lett 22, 1071 (1969).

[5]. B.S. DeWitt, Phys Rev 160, 1113 (1967).

[6]. C. Kiefer, Quantum Gravity (Oxford University Press, Oxford, 2007), $2^{\text {nd }}$ ed.

[7]. A. Riess et al., Astron J116, 1009 (1998).

[8]. A.O. Barvinsky and A.Y. Kamenshehik, Classical Quantum Gravity 7, L181 (1990).

[9]. A. Sen, J. High Energy Phys. 04 (2002) 048. 
[10]. C. Armendariz-Picon, V.F. Mukhanov and P.J. Steinhardt, Phys Rev D63, 103510 (2001).

[11]. M. Born and L. Infeld, Proc R Soc London, Ser A144, 425 (1934).

[12]. A. Frolov, L. Kofman, and A. Starobinsky, Phys Lett B545, 8 (2002).

[13]. I. Bars, S.H. Chen, and N. Turok, Phys Rev D84, 083513 (2011); I. Bars, S.H. Chen, P.J. Steinhardt, and N. Turok, arXiv : 1112.2470 .

[14]. R. Penrose, Structure of Space-Time (W.A. Benjamin, New York, 1970).

[15]. S.W. Hawking and R. Penrose, Proc R Soc A314, 529 (1970).

[16]. S.W. Hawking and G.F.R. Ellis, The Large Scale Structure of Space-Time (Cambridge University Press, Cambridge, 1973).

[17]. C.W. Misner, K.S. Thorne, and J.A. Wheeler, Gravitation (W.H. Freeman, San Francisco, 1973).

[18]. R. Brout and G. Venturi, Phys Rev D39, 2436 (1989); G. Venturi, Classical Quantum Gravity 7, 1075 (1990); C. Bertoni, F. Finelli, and G. Venturi, Classical Quantum Gravity 13, 2375 (1996).

[19]. A.Y. Kamenshchik, A. Tronconi, and G. Venturi, Phys Lett B702, 191 (2011).

[20]. A.A. Andrianov, F. Cannata, A.Y. Kamenshchik and D. Regoli, J. Cosmol. Astropart. Phys 02 (2008) 015.

[21]. A.B. Burd and J.D. Barrow, Nucl Phys B308, 929 (1988).

[22]. A.A. Starobinsky, Pis'ma Zh Eksp Teor Fiz 68, 721 (1988); [JETP Lett 68, 757 (1998)].

[23]. A. Balcerzak and M.P. Dabrowski, Phy Rev D73, 101301 (2006), Z. Keresztes, L.A. Gergely, and A.Y. Kamenshchik, arXiv : 1204.1199.

[24]. L. Fernandez-Jambrina and R. Lazkoz, Phys Rev D70, 121503 (2004).

[25]. M.P. Datrowski, T. Denkiewicz, and M.A. Hendry, Phys Rev D75, 123524 (2007).

[26]. Z. Keresztes, L.A. Gergely, V. Gorini, U. Moschella, and A.Y. Kamenshchik, Phys Rev D79, 083504 (2009).

[27]. Z. Keresztes, L.A. Gergely, A.Y. Kamenshchik, V. Gorini, and D. Polarski, Phys. Rev. D82, 123534 (2010).

[28]. A.O. Barvinsky, Phys Lett B241, 201 (1990); Phys Rep 230, 237 (1993).

[29]. S.J. Perlmutter et al., Astrophys J 517, 565 (1990).

[30]. A.A. Starobinsky, Gravitation Cosmol 6, 157 (2000).

[31]. R.R. Caldwell, Phys Lett B545, 23 (2002); Yu Shtanov and V. Sahni, Classical Quantum Gravity 19, L101 (2002).

[32]. A. Yu Kamenshchik, U. Moschella, and V. Pasoquier, Phys Lett B511, 265 (2001).

[33]. J.C. Fabris, S.V.B. Goncalves, and P.E. deSouza, Gen Relativ Gravit 34, 53 (2002); N. Bilic, G.B. Tapper, and R.D. Viollier, Phys Lett B535, 17 (2002); M.C. Bento, O. Bertolami, and A.A. Sen, Phys Rev D66, 043507 (2002).

[34]. V. Gorini, A.Y. Kamenshchik, and U. Moschelk, Phys Rev D67, 063509 (2003), T. Padmanabhan, Phys Rep 380, 235 (2003).

[35]. R.R. Caldwell, M. Kamionkowski, and N.N. Weinberg, Phys Rev Lett 91, 071301 (2003).

[36]. V. Gorini, A.Y. Kamenshchik, U. Moschella, and V. Posquier, Phys Rev D69, 123512 (2004).

[37]. J.D. Barrow, Classical Quantum Gravity 21, L79 (2004); 21, 5619 (2004); J.D. Barrow and C.G. Tsagas, Classical Quantum Gravity 22, 1563 (2005).

[38]. B. Carter, Phys Lett B224, 61 (1989); A. Vilenkin, Phys Rev D41, 3038 (1990).

[39]. A.Y. Kamenshchik, C. Kiefer, and B. Sandhofer, Phys Rev D76, 064032 (2007).

[40]. M. Bouhmadi-Lopez and P.V. Moniz, Phys Rev D71, 063521 (2005); M.P. Dabrowski, C. Kiefer, and B. Sandhofer, Phys Rev D74, 044022 (2006).

[41]. M. Bouhmadi-Lopez, C. Kiefer, B. Sandhofe and P.V. Moniz, Phys Rev D79, 124035 (2009).

[42]. N.D. Birrell and P.C.W. Davies, Quantum Fields in Curved Space (Cambridge University Press, Cambridge, U.K. (1984).

[43]. G.W. Gibbons and S.W. Hawking, Phys Rev D15, 2738 (1977).

[44]. J. Schwinger, Proc. Nett. Acad. Sci (U.S.A.) 37, 452 (1951); H.R. Lewis, Jr. and W.B. Riesenfeld, J. Math. Phys 10, 1458 (1969)

[45]. E. Mottola, Phys Rev D31, 754 (1985); B. Allen, Phys Rev D32, 3136 (1985); R. Bousso, A. Maloney and A. Strominger, Phys Rev D65, 104039 (2002) [hep-th/0112218]; E. Joung, J. Mourad and R. Parentani, JHEP 0608 (2006) 082 [hep-th/0606119]; JHEP 0709 (2007) 030 [arXiv : 0707, 2907].

[46]. L. Parker, Phys Rev 183, 1057 (1969); J. Haro and E. Elizalde, J Phys A Math Theor 41.372003 (2008); S.P. Kim, JHEP 0711 (2007) 048 [arXiv : 0710.0915]; J. Korean Phys Soc 531095 (2008) [arXiv : 0709.4313].

[47]. A.H. Guth and S.Y.Pi, Phys Rev D32, 1899 (1985); Sup Kim and C. Schubert, Phys Rev D84, 125028 (2011) [arXiv : 1110.0900]; S.P. Kini, H.K. Lee and Y. Yoon, Phys Rev D78, 105013 (2008) [arXiv : 0807.2696]. 